\title{
Simple, individually unique, and context-dependent learning methods for models of human category learning
}

\author{
TOSHIHIKO MATSUKA \\ Rutgers University, Newark, New Jersey
}

\begin{abstract}
The gradient descent optimization method has been a de facto standard learning algorithm in computational models of category learning. However, it can be considered as a normative (vs. descriptive) model of human learning processes. In particular, there are three concerns associated with the learning algorithm-namely, complexity, regularity, and context independency. In response to these limitations, the present study introduces an alternative, hypothesis-testing-like learning algorithm on the basis of a stochastic optimization method. The new learning model, termed SCODEL, provides qualitatively simple interpretations for its implied category-learning processes. Moreover, SCODEL is the first modeling attempt to depict individually unique and context-dependent learning processes. Four simulation studies were conducted and showed that the present model has the competence to operate as several different types of learners in various plausibly real-life situations.
\end{abstract}

The vast majority of recent neural network models of category learning-ALCOVE (Kruschke, 1992) for example-incorporate a form of gradient-based optimization methods as their learning algorithm. More precisely, these models incorporate an on-line version or stochastic approximation of gradient descent optimization as a learning algorithm, in which the model's coefficients are adjusted incrementally following the calculation of error for each individual training instance (Mitchell, 1997). This type of learning algorithm can be qualitatively interpreted as one in which humans try to learn about categories by adjusting all possible relevant knowledge about the categories (i.e., model coefficients - association weights and dimensional attention strength in the ALCOVE-type models) optimally in order to minimize misclassification whenever they receive feedback on their performance in categorization tasks. In other words, locally optimal changes are made incrementally and correctly, given the current stimulus instance, in the model's multifaceted coefficient space in every learning trial. Although this type of learning algorithm has been successfully applied to and replicated with many empirical, mainly aggregated data (e.g., Kruschke, 1992; Kruschke \& Johansen, 1999; Nosofsky, Gluck, Palmeri, McKinley, \& Glauthier,

This work was partly supported by the James McDonnell Foundation and the National Science Foundation under Grant EIA-0205178. I appreciate James E. Corter, Arieta Chouchourelou, Stephen Jose Hanson, Yasuaki Sakamoto, and anonymous reviewers for their helpful and constructive suggestions and comments. Correspondence concerning this article should be addressed to T. Matsuka, Howe School of Technology Management, Stevens Institute of Technology, Castle Point on Hudson, Hoboken, NJ 07030 (e-mail: tmatsuka@stevens.edu).
1994), its qualitative descriptions appear to be too normatively oriented for a model of ordinary individual learners. ${ }^{1}$ There are at least three concerns associated with such a learning algorithm.

The first concern is the implied complexity of the gradient descent algorithm applied to cognitive models. Although the human brain has very powerful computational competence, it seems implausible that people explicitly or consciously compute the gradient on each trial while attempting a classification task. At least, as a model of highorder conscious mind, the gradient descent's implied cognitive processes appear to be too complex. That is, the gradient descent type of learning seemingly illustrates a preconscious (or biological) level of learning processes, but not necessarily high-order conscious learning processes. The latter type of processes probably more closely describes human category learning. Even if people could find a proper updating direction for each coefficient, some researchers have raised a concern about the descriptive validity of gradual learning associated with gradient descent optimization. Bower and Trabasso (1963) have shown evidence that individuals learn a category in an all-or-none manner (i.e., classification accuracy suddenly increases from near base rate accuracy), which they referred to as insightful learning. In addition, Rehder and Hoffman (2003) observed characteristics of insightful learning not only in subjects' learning curves for categorization accuracy, but also in attention allocation. To put it in a more naive fashion, if people could find or believe they have found "correct" updating directions (i.e., gradient), why do they make the rather conservative adjustments that result in gradual learning? This concern may sound more persuasive if we realize that the calculation of a partial derivative can be rather computation- 
ally intensive (i.e., why do people put so much effort into making small changes?). Conceivably, this concern may be mere speculative guardedness, and humans' general cognitive processes and mechanisms might have evolved so as to approximate either first- or second-order gradient methods of optimization.

The second concern is the regularity of the gradient descent algorithms - that is, the gradient-based learning methods appear to allow a lesser degree of variability in learning trajectories at the individual level. Some realworld categories consist of many correlated and redundant feature dimensions (see Medin \& Schaffer, 1978). For this sort of category structure, it is very likely that there are several global and acceptable local minima in the error hypersurface. For example, Nosofsky, Palmeri, and McKinley (1994) showed that those who learned a training stimulus set successfully had differential responses to the novel stimuli, indicating that their successful learning might have resulted in different category knowledge (e.g., different attention weights). Similarly, Corter and Matsuka (2004) observed several qualitatively different attention distribution patterns among subjects who successfully learned the identical stimulus set, suggesting that multiple coefficient sets could have achieved successful classification (i.e., multiple minima). Gradient descent learning, however, has been shown to be incapable of replicating some illogical and/or less than optimal behaviors and learning processes of humans (Matsuka \& Corter, 2004). In other words, gradient-based learning often results in predictions of human cognitive processes that appear "too good to be true" in some cases. For example, when there are two perfectly redundant feature dimensions, the predicted learning curves of attention allocation for these two dimensions would be identical (or parallel, if initial attention weights are different) for gradient descent learning since it is being able to replicate only one possible qualitatively defined attention-learning trajectory-namely, the parallel utilization of the redundant dimensions. However, an empirical study showed that subjects learned to allocate different amounts of attention (operationally defined as the amount of time spent looking at a particular feature dimension) to perfectly redundant dimensions (Matsuka, 2002; Matsuka \& Corter, 2003). All the subjects exclusively or primarily paid attention to one dimension of two redundant dimensions (which apparently was selected randomly), but no single subject showed identical or parallel attentionlearning curves for the two redundant dimensions. In short, there are apparently some stochastic aspects in human cognitive processes, yet the many gradient-based learning methods do not sufficiently incorporate such stochasticity, resulting in a lesser degree of variability in learning trajectories and predicted behaviors. The learning algorithms, however, incorporate some stochastic elements - namely, the weak random association links between categories and exemplars or prototypes in the initial state of learning and the random stimuli presentation sequence. This can be interpreted as meaning that the many gradient-descent-type learning algorithms assume that individual differences in learning trajectories and acquired category knowledge can be explained perfectly only by prelearning mindsets about associations between categories and exemplars and by the order in which stimuli are introduced or encountered.

The last, but certainly not least significant concern is the context independency of virtually all learning algorithms that are based on the gradient descent. Human cognitive competence is often characterized by adaptability (e.g., Anderson, 1990), which manifests itself prominently in context-dependent thinking skills. But this type of cognitive ability has been largely overlooked in high-order cognitive-modeling research. In particular, virtually all models of human category learning assume that, in every instance, every learner has a rigid and $o p$ timal objective in any category-learning task-namely, error minimization. In other words, the models assume that everyone undertakes exactly the same computationally intensive operation for updating coefficients, irrespective of a learner's inter-, intra-, and extrapersonal contextual factors. This context independency is not specific to gradient-based optimization methods but is applicable to all learning algorithms. However, to account for multiple objectives in a category-learning task (e.g., error minimization and concept abstraction), the error function for the gradient-based optimization methods inevitably becomes more complex, because the objectives of learning need to be defined by multiple differentiable functions (see Matsuka, 2005). This, in turn, makes calculation of coefficient updates (e.g., partial derivatives) more computationally intensive. If we incorporate multiobjective operations into gradient-based learning, we certainly overcomplicate the cognitive processes implied by the model that are already complex (a detailed discussion is provided in the section on modeling).

These remarks are not intended to undermine the many neural network models of category learning with the gradient descent optimization. In fact, several models have successfully accounted for many empirical psychological phenomena and have provided valuable information leading to better understanding of the nature of category learning (e.g., Kruschke, 1992; Kruschke \& Johansen, 1999; Love, Medin, \& Gureckis, 2004; Nosofsky, Gluck, et al., 1994). However, the descriptive validity of the category-learning models with a standard gradient descent optimization method may be best attained at the aggregated group level. As models of individual learners, however, their learning algorithms' implied complexity, regularity, and context independency appear to be more normatively oriented, rather than describing how individuals process information when learning categories.

These limitations may be related to the fact that virtually all cognitive-modeling research has placed the greatest emphasis on feedforward algorithms, but substantially less on the descriptive validity of models' backward (i.e., learning) algorithms. With a belief that the learning algorithm for models of category learning is equally as im- 
portant as their feedforward algorithms, in the present study, a family of new, alternative learning algorithms is proposed, in which the emphasis is placed on capturing the qualitative essence of learning at the level of individual learners. The present alternative learning methods are intended to provide qualitatively simple interpretations of implied cognitive processes and to show variability and context dependency at the individual level. In particular, I apply, reinterpret, and extend Matsuka and Corter's (2004) stochastic learning models. The new learning algorithms proposed are general models of learning without any feedforward algorithm and do not restrict one to choose one network architecture over the other. In addition, because of their flexibility, the new learning methods can be applied to any quantitatively formulated models besides those of category learning. In the present study, the new leaning models are applied to ALCOVE, a model of category learning (Kruschke, 1992), because ALCOVE has been one of the most widely studied models of category learning and its qualitative descriptions of feedforward algorithm (i.e., categorization processes) are widely understood.

\section{ALCOVE}

Before describing the new model of learning in detail, ALCOVE (Kruschke, 1992), one of the most successful models of category learning, will be introduced as the particular example architecture selected in which to embed the new learning algorithm. For detailed descriptions and theoretical foundations of ALCOVE, readers are advised to refer to the original work by Kruschke. ALCOVE, for attention learning covering map, is a multilayer adaptive network model of categorization on the basis of the generalized context model (GCM; Nosofsky, 1986), a prominent exemplar model of classification. The first layer of ALCOVE is a stimulus input layer. Each node in this layer represents the value of the presented stimulus on a single dimension. Importantly, each dimension has an attention strength $\left(\alpha_{i}\right)$ associated with it. Usually, it is assumed that attention strengths are initially equal across dimensions, but the model learns to reallocate attention as learning proceeds. The next layer in the network is the exemplar layer. In a typical application, each node in this layer corresponds to an exemplar, described by its position in the multidimensional stimulus space, and receives input from relevant nodes in the input layer. The activation of each exemplar node is calculated on the basis of its similarity to the presented stimulus. ALCOVE uses a Minkowski $r$-metric formula (weighted by the dimensional attention strengths) in computing similarity. For example, for the city block metric where $r=1$, the activation of exemplar node $j$, denoted $h_{j}$, can be expressed as

$$
h_{j}=\exp \left[-c\left(\sum_{i} \alpha_{i}\left|\psi_{j i}-x_{i}\right|\right)\right],
$$

where $\psi_{j i}$ is the value of exemplar node $j$ on dimension $i$, $x_{i}$ is the activation of input node $i, c$ is a constant called specificity, and $\alpha_{i}$ is the attention strength for dimension $i$. In ALCOVE, the attention strengths essentially stretch and shrink dimensions: A high value of $\alpha_{i}$ will magnify any differences between the stimulus and the exemplar node values on that dimension.

The activity of the exemplar nodes is fed forward to the third layer, the category layer, whose nodes correspond to the categories being learned. The strength of association between category node $k$ and exemplar node $j$ is denoted by $w_{k j}$. The activation of category node $k$ is then computed as the sum of weighted activations of all exemplars, or

$$
O_{k}=\sum_{j} w_{k j} h_{j}
$$

The probability that a particular stimulus is classified as a member of Category $\mathrm{C}$, denoted as $P(\mathrm{C})$, is assumed to be equal to the activity of Category $\mathrm{C}$ relative to the summed activations of all the categories, where the activations are first transformed by the exponential function

$$
P(C)=\frac{\exp \left(\phi O_{c}\right)}{\sum_{k} \exp \left(\phi O_{k}\right)},
$$

where $\phi$ is a real-value mapping constant that controls the decisiveness of classification responses.

The standard ALCOVE uses a form of gradient descent for updating weights. The error term is defined as the sum of squared differences between the desired and the predicted outputs:

$$
E=\frac{1}{2} \sum_{k}\left(d_{k}-O_{k}\right)^{2}
$$

Partial derivatives of the error function with respect to the association weights $w_{k j}$ and the attention strengths $\alpha_{i}$ are obtained. Using the partial derivatives, the weight updates are obtained as follows:

$$
\Delta w_{k j}=-\lambda_{w} \frac{\partial E}{\partial w_{k j}}=\lambda_{w}\left(d_{k}-O_{k}\right) h_{j},
$$

and

$$
\begin{aligned}
\Delta \alpha_{i} & =-\lambda_{\alpha} \frac{\partial E}{\partial \alpha_{i}} \\
& =-\lambda_{\alpha} \sum_{j}\left[\sum_{k}\left(d_{k}-O_{k}\right) w_{k j}\right] h_{j} \cdot c\left|\psi_{j i}-x_{i}\right| .
\end{aligned}
$$

The $\lambda_{w}$ and $\lambda_{a}$ are the learning rates for the association weights and the attention strengths, respectively. The main goal of the present study is to provide an alternative learning algorithm that can be used as a replacement for the standard gradient descent learning incorporated into ALCOVE - namely, Equations 5 and 6-in order to offer simple, individually unique, and context-dependent learning processes. 


\section{LEARNING BY HYPOTHESIS TESTING}

\section{General Model}

The present proposed learning model is termed SCODEL (for stochastic context dependent learning model) and is on the basis of Matsuka and Corter's (2004) stochastic learning model and on the subsequent biased stochastic learning model (Matsuka, 2004) both of which are founded on simulated annealing optimization methods (see, e.g., Ingber, 1989; Metropolis, Rosenbluth, Rosenbluth, Teller, \& Teller, 1953). The fundamental mechanism of SCODEL is rather simple: SCODEL (quasi)randomly generates a set of hypotheses about the properties of the categories being learned and keeps or discards the hypotheses, depending on how good they are.

In SCODEL, people are assumed to form a hypothesis about category structures in quasirandom fashion. The hypothesis in the present framework is directly related to the network architecture or coefficients of a forward model (i.e., ALCOVE in the present study). Hypothesis testing is often used to describe the explicit (e.g., conscious or verbalizable) learning processes for categories (Ashby, Alfonso-Reese, Turken, \& Waldron, 1998; Love, 2003). With reference to an ALCOVE-type feedforward model, at the beginning of each training epoch, on the basis of the currently most successful hypotheses set, SCODEL quasirandomly produces hypotheses about the relationship between exemplars and category membership (i.e., association weights), along with hypotheses about the diagnosticities of the feature dimensions that determine the distribution of attention (i.e., attention strengths). This is accomplished by randomly updating each coefficient with an independently sampled term from a prespecified, in general, zero-mean symmetric distribution (e.g., the Gaussian distribution). Thus, Equations 4 and 5 are replaced by the following functions:

$$
\begin{gathered}
w_{k j}^{t}=w_{k j}^{s}+\Delta w_{k j}, \\
\Delta w_{k j} \sim \Phi^{w}\left(T^{t}\right), \\
\alpha_{i}^{t}=\alpha_{i}^{s}+\Delta \alpha_{i},
\end{gathered}
$$

and

$$
\Delta \alpha_{i} \sim \Phi^{\alpha}\left(T^{t}\right),
$$

where the superscript $s$ indicates previously accepted coefficient values, $\Delta w$ and $\Delta \alpha$ are random numbers generated from prespecified distributions $\Phi^{w}$ and $\Phi^{\alpha}$, which take a value called temperature (see Metropolis et al., 1953) at time $t$. The temperature decreases across training blocks according to the following annealing schedule function:

$$
T^{t}=\delta(v, t) \text {, }
$$

where $\delta$ is the temperature-decreasing function that takes the temperature-decreasing rate $(v)$ and time $(t)$ as inputs. A choice of the annealing function (i.e., Equation 11) may depend on the particular choice of the random number generation functions, or Equations 8 and 10 (Ingber, 1989). Note that the decrease in the temperature causes decreases in the width of the distributions $\Phi^{w}$ and $\Phi^{\alpha}$.

The new sets of coefficients ( $w_{k j}^{t}$ and $\alpha_{i}^{t}$ ) are updated on the basis of the currently accepted coefficients $\left(w_{k j}^{s}\right.$ and $\alpha_{i}^{s}$ ), and thus Equations 7 and 9 indicate that people use the currently accepted hypothesis as a basis for generating a new hypothesis. Thus, the present model does not assume that learning is carried out by totally stochastic processes; rather, it assumes that people utilize their experience with stimuli from given categories. The effect of transition in distribution widths controlled by temperature can be interpreted as follows. In the early stages of learning, SCODEL is quite likely to produce "radical" hypotheses (i.e., the new set of hypotheses, and thus coefficients, is very different from the currently valid and accepted hypotheses). But as learning progresses, the widths of the random distribution decrease, so that it increasingly stabilizes its hypotheses and establishes more concrete knowledge of the category.

The set of hypotheses (i.e., the set of new coefficient values) is then accepted or rejected, on the basis of the computed relative utility (defined below) of the new hypothesis set. Note that in the SCODEL framework, a smaller utility value indicates better utility. Specifically, if the new coefficient values result in better utility (i.e., meeting learners' objectives better), they are accepted. If they result in poorer utility, they are accepted with some probability $P$, defined by the relative utility of the new hypotheses against that of the currently accepted hypotheses. In a general form, the probability of accepting a new set of coefficients is defined as is shown below if $Z\left(\boldsymbol{w}^{s}+\Delta \mathbf{w}, \boldsymbol{\alpha}^{s}+\Delta \boldsymbol{\alpha}\right)>Z\left(\mathbf{w}^{s}, \boldsymbol{\alpha}^{s}\right)$, or as 1 otherwise, where $Z\left(\mathbf{w}^{s}+\Delta \mathbf{w}, \boldsymbol{\alpha}^{s}+\Delta \boldsymbol{\alpha}\right)$ is a function defining the utility of the new coefficient set, $Z\left(\mathbf{w}^{s}, \boldsymbol{\alpha}^{s}\right)$ is the utility of the previously accepted set, and $T^{t}$ is the temperature at time $t$. When accepted, the accepted coefficients will be replaced by the current coefficients. Equation 12 indicates that the acceptance of hypotheses is influenced largely by the definition of the utility of the coefficient set.

In sum, SCODEL does not assume that the learning of categories involves computationally intensive (back) propagations of classification error in the multifaced coefficient space or calculation of partial derivative for each coefficient for the error hypersurface. Rather, in the present learning model framework, a very simple operation (e.g., comparison of two utility values), along with the operation of stochastic processes, is assumed to be the key mechanism in learning. In SCODEL, knowledge about the category evolves as learning progresses by permitting,

$$
P\left(\Delta \mathbf{w}, \Delta \boldsymbol{\alpha} \mid \mathbf{w}^{s}, \boldsymbol{\alpha}^{s}, T\right)=\left\{1+\exp \left[\frac{\mathrm{Z}\left(\mathbf{w}^{s}+\Delta \mathbf{w}, \boldsymbol{\alpha}^{s}+\Delta \boldsymbol{\alpha}\right)-\mathrm{Z}\left(\mathbf{w}^{s}, \boldsymbol{\alpha}^{s}\right)}{T^{t}}\right]\right\}^{-1}
$$


mainly, "good" sets of hypotheses and, occasionally, "bad" ones to survive and by using such enduring hypotheses as bases for generating a new set of hypotheses. The goodness or badness of a set of hypotheses in the SCODEL framework is purposely designed to be situation specific, resulting in context-dependent learning processes. In the next section, several different utilities of hypotheses will be discussed in detail.

\section{Utilities of Hypotheses}

One important element of SCODEL is that the same set of hypotheses (e.g., a set of coefficients, $\mathbf{w}$ and $\boldsymbol{\alpha}$ ) for the same categories can have very different utility values, depending on the situational characteristics. In particular, in the SCODEL framework, one can take into account context-dependent cognitive processes by using the utility function, made up of multiple functions. In this section, several illustrative context-dependent objectives associated with category learning will be outlined, along with possible functions quantifying such objectives.

Error minimization (EMN) function. Here, the objective of learning is defined strictly on the basis of categorization error in the training stimulus set, without any influences from any contextual factors. In other words, concept formation on the basis of this particular objective is driven solely by error minimization via a hypothesistesting-like stochastic optimization process. People may have this type of objective (perhaps unintentionally) when the cognitive resources required for a categorization task are small or (most likely, intentionally) when there is a need for explicitly allocating a large amount of cognitive resources in order to minimize categorization error (e.g., preparing an exam for an introductory psychology course).

Operationally, the sum of squared error is an example of such an objective, and thus the utility function $Z(\cdot)$ can be formulated as

$$
\mathrm{Z}(\theta)=L(\theta)=\sum_{n=1}^{N} \sum_{k=1}^{K}\left(d_{k}-O_{k}\right)^{2}
$$

where $w_{k j}, \alpha_{i} \in \theta, K$ is the number of categories, $N$ is the number of input stimuli in one block, $d_{k}$ is a desired output (i.e., correct category) for category node $k$, and $O_{k}$ is the network's predicted categories (see Equation 2). This utility function is qualitatively identical to the majority of category-learning models, including ALCOVE. The only difference is that ALCOVE estimates the classification error with only a single instance, whereas SCODEL obtains the estimate on the basis of multiple instances, due to a general tendency for SCODEL to require a more stable estimate of classification accuracy of a particular hypotheses set. Although this error minimization objective is almost identical to the standard ALCOVE's objective in learning (Equation 4), due to its stochastic learning process SCODEL could result in qualitatively different learning outcomes than ALCOVE does, as shown in simulation studies.
Efficiency and abstraction (ABS) function. Strict error minimization can and often does result in overgeneralization and ineffective usage of resources, such as memory. The human brain has a very powerful computational power, but in reality, as has empirically been suggested (Corter \& Matsuka, 2004; Matsuka, 2002; Matsuka \& Corter, 2003), we often do not put every possible effort into everyday categorization tasks. Rather, we usually, perhaps implicitly, try to minimize categorization error with a smaller amount of effort or try to have manageably simple, acceptably accurate category knowledge, balancing accuracy and simplicity. A psychological phenomenon called the basic-level category (Rosch, Mervis, Gray, Johnson, \& Boyes-Braem, 1976) may be associated with this balanced, manageably simple, yet acceptably accurate, categorization. That is, people have a tendency to categorize stimuli at the middle level of abstraction, resulting in manageably simple concepts. In addition, people have been shown to be more accurate or quicker in categorizing at the basic level than at other levels (Kosslyn, Alpert, \& Thompson, 1995; Rosch etal., 1976). This tendency to categorize stimuli at the basic level might be a reflection of the inherent human preference for having category structures that are manageably simple, yet sufficiently accurate. Thus, efficiency (in terms of cognitive resources, but not necessarily computational speed) and simplicity appear to be our objectives in the formation of some concepts or, in a similar manner, in category-learning tasks. Note that simplicity in categorization can be interpreted as the abstraction of concepts, making the stimulus space manageably compact, which in turn makes it readily available and accessible for other, high-level cognitive processes.

In this type of objective, the utility of a particular coefficient configuration can be defined as a weighted sum of the accuracy in classification and the mental effort required by the coefficient configuration. Thus, the utilitydefining function consists of two independent indicesnamely classification accuracy $(L)$ and mental effort $(Q)$, both dependent on learnable coefficients $\mathbf{w}$ and $\boldsymbol{\alpha}$ at time $t$ and formulated as

$$
Z(\theta)=L(\theta)+Q(\theta)
$$

where $L(\theta)$ is defined as in Equation 13. The $Q$ function may be considered as a penalty function, penalizing "complex" coefficient configurations that are believed to require more mental effort. The general form of $Q$ function is given as follows:

$$
Q(\theta)=\gamma_{w} \Omega(w)+\gamma_{\alpha} A(\alpha),
$$

where $\Omega$ and $A$ are functions indicating the compactness or abstractness of the current category knowledge (i.e., coefficient configuration at time $t, \mathbf{w}^{t}$ and $\boldsymbol{\alpha}^{t}$ ) that is associated with the mental effort required for the specific coefficient configurations. Two scalars, $\gamma_{w}$ and $\gamma_{\alpha}$, are coefficients weighting these mental efforts. Note that $\gamma_{w}$ and $\gamma_{\alpha}$ also control the relative importance of $L$ and $Q$ 
functions (i.e., accuracy vs. simplicity). That is the hypothetical coefficient, $\gamma_{Q}$, weighting the importance of $Q$ function relative to the $L$ function, is included in $\gamma_{w}$ and $\gamma_{\alpha}$. That is, $\gamma_{w}=\gamma_{Q} \gamma_{w}$, and $\gamma_{\alpha}=\gamma_{Q} \gamma_{\alpha}$. Thus Equations 14 and 15 may be rewritten as

$$
Z(\theta)=L(\theta)+\gamma_{Q} Q^{*}(\theta)
$$

and

$$
\gamma_{Q} Q^{*}(\theta)=\gamma_{Q} \gamma_{w}^{*} \Omega(w)+\gamma_{Q} \gamma_{\alpha}^{*} A(\alpha) .
$$

There are several functions applicable for $\Omega$ and $A$ :

$$
\begin{gathered}
\Omega(w)=\sum_{j} \sum_{k} w_{k j}^{2}, \\
A(\alpha)=\sum_{i} \alpha_{i}^{2}, \\
\Omega(w)=\sum_{j} \sum_{k} I\left(\left|w_{k j}\right|>\zeta^{w}\right),
\end{gathered}
$$

and

$$
A(\alpha)=\sum_{i} I\left(\alpha_{i}>\zeta^{\alpha}\right),
$$

where $\zeta^{w}$ and $\zeta^{\alpha}$ are threshold values, and $I$ (expression) is the indicator function that returns 1 if the expression is satisfied. Equation 18, often referred to as the ridge penalty function or weight decay, encourages coefficient settings that have small coefficient values, whereas Equation 19 encourages coefficient settings that have a large number of coefficients with less than the threshold values, $\zeta$ s. More general functions for $\Omega$ and $A$ are given as follows:

$$
\Omega(w)=\sum_{j} \sum_{k} \frac{\left(\frac{w_{k j}}{q}\right)^{2}}{1+\left(\frac{w_{k j}}{q}\right)^{2}}
$$

and

$$
A(\alpha)=\sum_{i} \frac{\left(\frac{\alpha_{i}}{q}\right)^{2}}{1+\left(\frac{\alpha_{i}}{q}\right)^{2}},
$$

where $q$, which can be either time dependent or independent, controls the types of penalization or encouragement. That is, Equation 20 approaches Equation 18 as $q \rightarrow \infty$ and approaches Equation 19 (with $\zeta=0$ ) as $q \rightarrow$ 0 (Cherkassky \& Mulier, 1997).

In many simulation studies, relative, but not absolute, predicted attention allocation strengths are analyzed and compared (Matsuka, 2002; Matsuka, Corter, \& Markman, 2003). In such cases, the relative attention strengths $a_{i}=$ $\alpha_{i} / \Sigma_{m}\left(\alpha_{m}\right)$ should be used as inputs for the penalty function. In addition, the penalization functions do not have to be in the same form for association weights and attention strengths.
Domain expertise. Having a compact category structure often reduces the memory load and the extent of other cognitive effort, which in turn may allow humans to have fast and acceptably accurate categorizations. Again, this sort of process may be exemplified by the characteristics of the basic level of categorization - that is, balanced simplistic accurate category knowledge helps us make effective usage of categories, such as in communication and reasoning. However, in some situations, people are often able to recognize many exemplars in a particular category population and are less likely to confuse one exemplar with another (Tanaka \& Gauthier, 1997). In addition, it has been shown that it is possible to acquire domain expertise even in experimental settings as the experimenters systematically manipulate the level of category hierarchy (Johnson \& Mervis, 1997; Tanaka \& Taylor, 1991). For those domain experts, the ability to acknowledge each exemplar may be an essential objective, but such an outcome seems less likely to be expected if the utility function is on the basis of strict error minimization (see the Error Minimization section above) or concept abstraction (the ABS function). Here, the exemplar recognition or expertise index for a particular set of hypotheses is defined qualitatively as how confusable the maximally responded exemplar unit is, as compared with other exemplars, and the index is defined operationally as the ratio of the activation of the maximally responded exemplar unit (summed over one training block) to that of the second maximally responded exemplar unit:

$$
\Gamma(\theta)=\Gamma(\alpha)=\sum_{n=1}^{N}\left(\frac{h_{\max -1}}{h_{\max }}\right),
$$

where $h$ is the activity of the exemplar units (Equation 1), $N$ is the number of input stimuli in one training block, and the subscripts max and max -1 indicate the exemplar unit with the maximal and the second maximal activations, respectively. Note that this index is affected only by the dimensional attention coefficients, because the activations of the exemplar units are a function of attention strength, but not of association weights. Therefore, it takes only attention coefficients, $\alpha$.

The $\Gamma$ function encourages learners to pay attention to feature dimensions that help discriminate among exemplars in their memory during recognition. As with the ABS function in SCODEL, the utility function $Z$ for this type of objective consists of two independent functions, categorization accuracy $(L)$ and the expertise index $(\Gamma)$ :

$$
Z(\theta)=L(\theta)+\gamma \Gamma(\theta)
$$

where $\gamma$ is a coefficient for the expertise (recognition) index. This makes the learner a domain expert who is knowledgeable about many specific exemplars within a particular category domain with correct category membership.

Prediction in a nonperfect world (ATT). It is believed that a more compact model (or concept) tends to offer better generalizable predictions or is less likely to result in overgeneralization. In this regard, having an ob- 
jective of acquiring compact category knowledge (i.e., the ABS function) may provide sufficient predictive potential without overgeneralizing what is learned. However, in real-world categorization problems, we sometimes do not have access to all the information. That is, there are many cases in which information about some feature dimensions is missing or is prohibitively expensive (physically, economically, and/or psychologically) to obtain. It seems more effective to be conservative in terms of cognitive efficiency and to allocate attention to many, if not all, feature dimensions in order to prepare for situations in which some feature dimensions are missing or untenable. For example, a good stock market investor who learns to pay attention to a greater number of feature dimensions probably has a better chance of correctly predicting the category of a particular stock's prospective (e.g., good or bad) than investors who pay attention to a smaller number of feature dimensions have, particularly when they could not access information on some diagnostic feature dimensions. However, in order to have more generalizable knowledge, experience with some specific exemplar stock should be regularized to some extent. In other words, the association weights between exemplar units and category nodes need to be somehow regularized, as in Equation 17. In this regard, this type of objective resembles the efficiency objective (Equation 17), but is distinct from the objective expressed in Equation 17 in that paying attention to more feature dimensions is encouraged, but not discouraged, in the present set. Thus, here, an operational definition can be constructed by small modification of that of concept abstraction (ABS). In particular, the utility function may be defined as

$$
Z(\theta)=L(\theta)+\gamma_{w} \Omega(w)+\gamma_{\alpha} A^{*}(\alpha)
$$

where, $L$ (i.e., classification accuracy) and $\Omega$ (i.e., complexity regularization) are defined as in ABS section. The $A^{*}$ function can be formulated as

$$
A^{*}(\alpha)=\sum_{i} I\left(\alpha_{i}<\zeta^{\alpha}\right),
$$

which is the opposite of Equation 19, in that it encourages the coefficient configuration with a large number of (relative) attention coefficients greater than the threshold value $\zeta^{\alpha}$.

Communicative common concepts (COM). Many natural categories are often defined by highly correlated and redundant feature dimensions (Medin \& Schaffer, 1978). For such categories, there are several ways to achieve error minimization or utility maximization, as has empirically been suggested (Corter \& Matsuka, 2004; Nosofsky, Gluck, et al., 1994). In other words, various coefficient configurations or concepts can result in similarly effective utilities. However, if different people have different ideas or knowledge about a category, it seems very difficult, if not impossible, for them to effectively communicate about the category. As several cognitive scientists have suggested, one of the most important functions and operations of categorization is to enhance the flow of communication (e.g., Markman \& Gentner, 1996). Then, it appears necessary to some extent for individuals to adjust their knowledge about particular categories in order to make the categories communicative, an important utility of categorization empirically suggested by Markman and Makin (1998). Thus, we probably have an implicit or explicit objective to learn to form category structures that have an acceptable level of norm or commonality within a defined group.

Here, the communicative index, which is intended to capture commonality of category, is defined qualitatively as how much a set of hypotheses about a category resembles a criterion set of category structures. Operationally, the communicative index is defined by the sum of the squared differences between coefficient configuration/ values (relative values for $\alpha \mathrm{s}$ ) of the criterion and those of the learner, or

$$
\begin{aligned}
\Pi(\theta)= & \gamma_{w} \sum_{k}^{K} \sum_{j}^{J}\left(w_{k j}^{*}-w_{k j}\right)^{2} \\
& +\gamma_{\alpha} \sum_{i}^{I}\left(\frac{\alpha_{i}^{*}}{\sum_{m=1}^{I} \alpha_{m}^{*}}-\frac{\alpha_{i}}{\sum_{m=1}^{I} \alpha_{m}}\right)^{2},
\end{aligned}
$$

where the asterisk $(*)$ indicate the criterion.

The word criterion does not necessarily mean correct or accurate. Moreover, the criterion coefficient configuration does not need to be defined a priori. Rather, it can be changed dynamically. For example, when there are two learners, each one of them can be a criterion for the other while they are learning the category. For stimuli defined by many feature dimensions and/or many exemplars, correlation coefficients may be used as the $\Pi$ function, instead of the sum of squared differences. This type of objective can be combined with other objective types. For example, within a domain expert community, the criterion coefficient configuration should incorporate the expertise index (Equation 21), whereas within a conservative or careful learners' community, Equation 23 should be employed in combination with the communicative index.

It is clear that this operational definition is overly simplified and/or overly stringent, in that it compares all coefficient values at once. However, it may capture the essence of cooperative learning sufficiently.

\section{Remarks on SCODEL}

SCODEL is devised to depict simple, individually different, and context-dependent learning processes in category formation. As has been shown above, although SCODEL's fundamental processes are fairly simple, its computational implementation (i.e., computer program) is not always simpler than a standard gradient descent optimization method. However, the model's implied processes are qualitatively simpler and potentially more descriptive than calculation of partial derivatives is as a model of humans' learning processes. As a descriptively 
oriented model of human cognition, SCODEL places stronger emphasis on the qualitative description of depicted processes than on quantitative procedures operating somewhat in a "black box" manner. In this regard, the present learning model may have plausibly captured the essence of simple cognitive process in category learning.

In the SCODEL framework, each coefficient is updated by an independently drawn random number, and thus, it is very unlikely to have parallel utilization of two redundant dimensions (but it probably could have nearly parallel utilization, depending on situational constraints or learning objectives). Rather, it would predict large individual differences, depending on the learners' objectives and/or stochasticity in learning. In other words, the present models can result in numerous patterns of exemplar-category association strengths and numerous patterns of attention allocation to feature dimensions, as long as the coefficient configurations result in the acceptable level of utility defined by contextual factors. This, in turn, may lead to "superficial" beliefs that some irrelevant dimensions are somewhat relevant to particular categories, apparently a common phenomenon in ordinary life (e.g., believing in a jinx). Thus, when there are several minima, which is probably true for real-world category-learning tasks, stochastic learning can result in several different learning trajectories and coefficient configurations, corresponding to possible individual differences. This characteristic seems important, because several empirical studies of category learning have reported such individual differences (Matsuka \& Corter, 2003; Nosofsky, Palmeri, \& McKinley, 1994).

To achieve situationally influenced context-dependent cognitive processes, SCODEL incorporates a flexible utility function, which is often defined by more than one additive function, accounting for multiple objectives and contextual factors in learning processes, such as minimization of classification error and reduction in concept complexity. In many applications, SCODEL would incorporate a complexity-controlling process, since it is generally expected that humans prefer acceptably accurate simple concepts over marginally superior but complex concepts (e.g., Feldman, 2003). In that respect, SCODEL can be considered as a type of regularized-learning model. But its regularization function, illustrated above, does not necessarily result in a compact coefficient configuration, and in this regard, SCODEL is different from a standard regularization method in the machine-learning literature (see, e.g., Haykin, 1999). Rather, SCODEL's implied regularization processes are intended to be heuristically oriented in order to enhance qualitative interpretability of its depicted cognitive processes and to plausibly describe conscious cognitive processes at (near) their face values. There are many other applicable and, perhaps, more sophisticated ways to define various objectives, situational constraints, and/or motivations. However, as an initial attempt, the utility functions discussed in the present article may capture the essence of sufficiently broad areas of context-dependent learning processes adequately.

Complexity in learning. SCODEL is developed to model simple learning processes, yet its context dependency processes may appear to be rather complex. However, its implied cognitive processes can be interpreted as, in general, simpler than that of the gradient descent leaning method. For example, if one disregards the computational complexity of random number generation (i.e., by assuming that random number generation requires very little mental effort), SCODEL calculates only $M^{w+\alpha}+$ $M^{w}+M^{\alpha}$ number of values in learning, where $M^{w+\alpha}, M^{w}$, $M^{\alpha}$ are the number of contextual factors defined for both association weights and attention, association weights only, and attention only, respectively. For example, for a learner who prefers to have compact concepts (i.e., the ABS function), SCODEL calculates only three independent values in each step of learning, regardless of the number of exemplars and the dimensionality of the stimulus set. In contrast, in each learning step, the gradientdescent-based model with the same contextual factor requires calculations of $M^{w+\alpha} \times(J \times K+I)+M^{w} \times J \times$ $K+M^{\alpha} \times I$ coefficient updates, where $J, K$, and $I$ are the numbers of exemplars, category types, and feature dimensions, respectively. Thus, for a two-category, fourdimension, 10-exemplar stimulus set, there would be $24+$ $20+4=48$ values that need to be calculated for an abstractive learner in every learning instance. Even if one cannot disregard the computational complexity of random number generation, its required mental effort appears to be much less than that for the gradient descent. That is, a gradient-descent-based learning model assumes that all coefficient updates will be executed correctly, defined with some complex mathematical operations (e.g., Equations 5 and 6), whereas SCODEL's stochastic hypothesis generation does not assume any correctness, nor is it restricted by mathematical operations.

\section{SIMULATIONS}

In order to investigate the capabilities of the SCODEL framework to operate as different types of hypothetical, yet plausible, learners do and in order to replicate human category-learning processes, four simulation studies were conducted. The present series of simulation studies did not emphasize SCODEL's accuracies in learning per se. Instead, greater emphasis was placed on its capabilities in showing different expectable context-dependent learning by hypothetical people engaged in hypothetical category-learning tasks, with the aim of evaluating its potential as an alternative model of high-order learning processes in humans. In one simulation study, however, SCODEL's prediction and empirical data for classification accuracies and attention processes were compared.

In the first simulation study, the effect of SCODEL's objective functions on attention coefficients was investigated. In Simulation 2, the simulated subjects were trained via corrective feedback and communicating with other simulated subjects. Simulation3, on the other hand, 
Table 1

The Abstract Category Structure Used in Simulation Studies 1 and 2

\begin{tabular}{cccccc}
\multicolumn{7}{c}{ Simulation Studies 1 and 2 } \\
\cline { 2 - 6 } Category & 1 & 2 & 3 & 4 & 5 \\
\hline A & 0 & 0 & 0 & 1 & 1 \\
A & 0 & 0 & 0 & 1 & 2 \\
A & 0 & 0 & 1 & 0 & 3 \\
A & 0 & 0 & 1 & 0 & 4 \\
B & 1 & 1 & 0 & 1 & 5 \\
B & 1 & 1 & 0 & 1 & 6 \\
B & 1 & 1 & 1 & 0 & 7 \\
B & 1 & 1 & 1 & 0 & 8 \\
\hline
\end{tabular}

was intended to show that a type of SCODEL could partially overcome a limitation of the exemplar theory. In the final simulation study, the predictions of SCODEL were compared with an empirical study in order to show its advantage over the standard gradient descent optimization methods as a descriptive model of human category learning.

\section{Simulation 1: Individual Learners With Different Objectives}

The effect of SCODEL's context-dependent learning on its attention coefficients was investigated in the present simulation study.

Methods. The stimulus set shown in Table 1 was used in Simulation 1. It should be noted that all feature values in Dimension 5 were treated as nominal values differentiating each element within the dimension, and thus their numeric differences do not have any meaning. Note that this stimulus set is very simple to categorize and that there are several possible attention distribution patterns in which ALCOVE with the SCODEL algorithm can achieve successful learning. Dimensions 1, 2, and 5 were solely diagnostic, but any combinations that included Dimension 1, 2, or 5 could result in perfect categorization performance.

There were three types of context-dependent learners involved in the present simulation study-namely, $a b$ stractive learners, attentive learners, and domain experts. The abstractive learners are those whose objective is to learn an abstract representation of the stimulus set. This type of learner is expected to pay attention to either Dimension 1 or 2 but is less likely to pay attention to both. The attentive learners, on the other hand, are those who try to pay attention to all the feature dimensions. The domain experts, whose objective is to have coefficient configurations that help them to recognize all exemplars, are expected to pay attention to Dimension 5, which consists of all the unique feature values.

For all three types of learners, random numbers drawn from the Cauchy distribution were used for updating both association weights and attention strengths. The random number generation function was on the basis of Ingber (1989). The temperature was exponentially decreased during a learning epoch, on the basis of the function suggested by Ingber. For the abstractive learner, the decay function (Equation 18A) was used to penalize association weights, and the subset selection function (Equation 19B with $\zeta=0.15$ ) was used for attention strengths. For the attentive learners, the decay function was used for association weights, and the subset selection function (Equation 24 with $\zeta=0.15$ ) was used for attention strengths. As in Equation 22, the utility function for the experts was defined by two independent functions - namely, error minimization (Equation 13) and the expertise index (Equation 21). The $\gamma$ coefficients were selected arbitrarily $\left(\gamma^{w}=0.1\right.$ and $\gamma^{\alpha}=0.5$ for abstractive learners; $\gamma^{w}=0.2$ and $\gamma^{\alpha}=0.5$ for attentive learners; $\gamma=1.5$ for domain experts). ALCOVE's specificity parameter $c$ and decisiveness parameter $\phi$ were set to 1 and 3, respectively, for all three types of learners (see Equations 1 and 3). They were run in a simulated training procedure in which the correct classification responses were learned for the training set. For all types of learners, there were 100 simulated subjects trained with 500 training blocks.

Results. Table 2 shows mean classification errors and dimensional attention weights for the three types of learners. All three types of learners successfully learned to categorize the stimuli. Note that the classification error does not have much of a significant meaning here, because it can be easily altered by manipulation of ALCOVE's decisiveness parameter $\phi$ in Equation 3. As was expected, the abstractive learners, on average, paid attention to Dimensions 1 and 2. However, as is shown in Figure 1A, the majority of simulated subjects paid attention to either Dimension 1 or Dimension 2, and only $13 \%$ of simulated subjects paid more than $15 \%$ (i.e., threshold) of their attention to both Dimensions 1 and 2 . In contrast, the attentive learners paid attention to all the feature dimensions (with a stronger emphasis on Dimensions 1 and 2, however). Unlike the abstractive learners, the attentive learners paid similar amount of attention to Dimensions 1 and 2 (Figure 1B). Both abstractive and attentive learners showed the differentiated utilization of the redundant feature dimensions (i.e., Dimensions 1 and 2). For the domain experts - again, as was expected - the simulated subjects paid attention to Dimension 5, which provided discriminatory information about different exemplars.

Discussion. In the present simulation study, three types of simulated subjects in a hypothetical categorylearning task were successfully reproduced by SCODEL with three different context-dependent objectives. Standard gradient descent learning can result in attentive

Table 2

Results of Simulation Study 1 for Three Types of Hypothetical Learners

\begin{tabular}{lcccccc}
\hline & & \multicolumn{5}{c}{ Attention Strengths } \\
\cline { 3 - 7 } Type of Learner & Error & D1 & D2 & D4 & D4 & D5 \\
\hline Abstractive & 0.116 & 0.304 & 0.308 & 0.125 & 0.124 & 0.140 \\
Attentive & 0.100 & 0.265 & 0.266 & 0.161 & 0.161 & 0.147 \\
Expertise & 0.064 & 0.223 & 0.197 & 0.089 & 0.079 & 0.411 \\
\hline
\end{tabular}



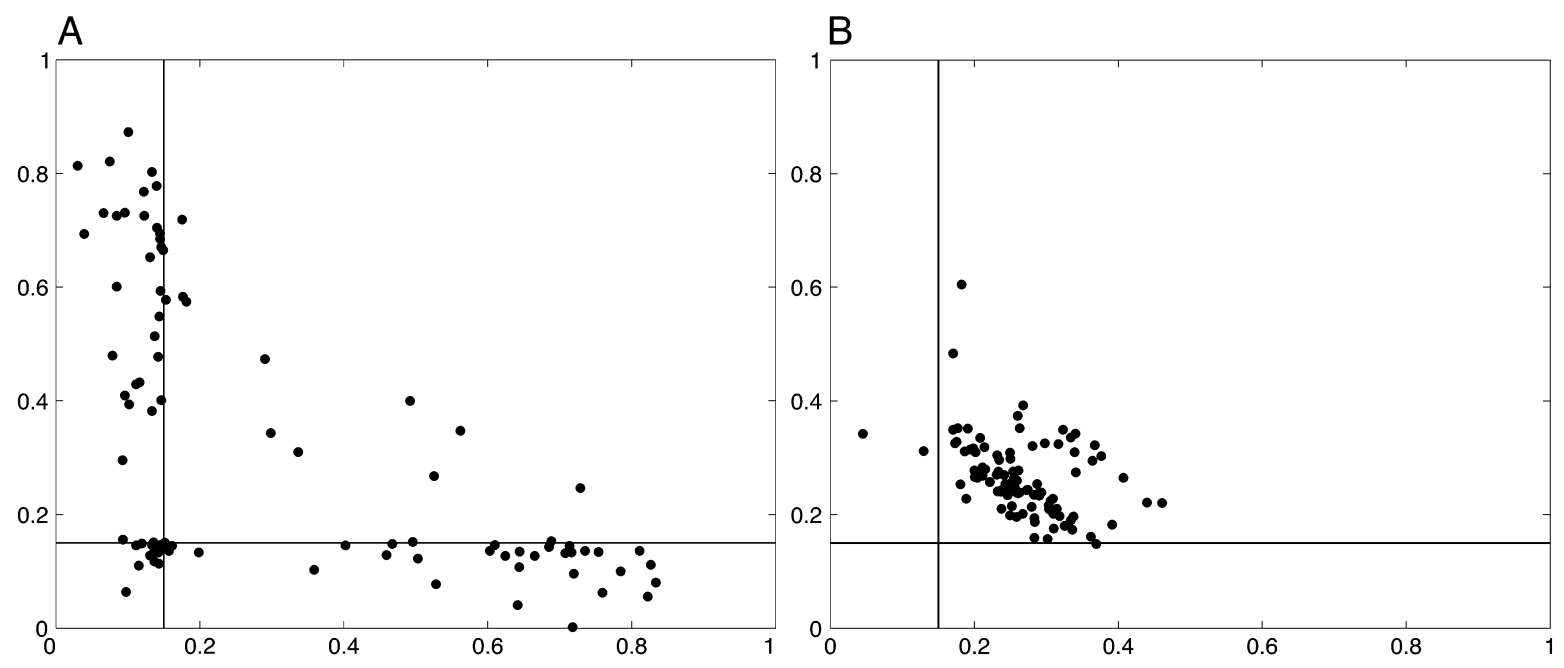

Figure 1. Results of Simulation 1. (A) Relative attention allocated to Dimension 1 ( $x$-axis) versus 2 ( $y$-axis) for the abstractive learners. (B) Relative attention allocated to Dimension 1 versus Dimension 2 for the attentive learners. The vertical and horizontal lines indicate the threshold values.

learners and experts, depending on parameter settings (Matsuka, Corter, \& Hanson, 2004). For example, attentive learners can be achieved with a zero learning rate for attention. However, such parameter arrangement qualitatively implies that the simulated learner is incapable, unmotivated, and/or unwilling to learn to selectively allocate his or her attention. In reality, however, there may be a case in which a learner is capable, motivated, and willing to selectively pay attention but decides to allocate attention evenly in order to take a conservative approach. For this type of learner, the standard ALCOVE may replicate its behavioral patterns without qualitatively corresponding cognitive processes or parameters, because its learning algorithm - namely, gradient descent - in its current form, does not emphasize context-dependent learning.

It may not be apparent at first glance, but the present simulation study also showed that SCODEL is capable of reproducing more realistic individual differences in attention processes than the gradient-based learning is. That is, when there are two (or more) perfectly redundant dimensions, the standard gradient descent learning method would always allocate exactly the same amount of attention to those dimensions. Such a prediction appears clearly to be unrealistic. To illustrate this unrealistic prediction, a supplementary simulation study was conducted in which the standard gradient-based ALCOVE was used with randomly selected parameter values. In particular, 10,000 simulated ALCOVE subjects were trained to categorize the same stimulus set. The ranges of parameters were [0.1 10] for $c$ and $\phi$ and [0.001 1] for the two learning rates. Table 3 shows the results of the supplemental simulation study, along with those from Simulation 1. In particular, it shows the squared differences in the amount of attention allocated to Feature Dimensions 1 and 2. As was claimed, the standard gradient descent algorithm always paid exactly the same amount of attention to the redundant dimensions, regardless of how quickly or slowly the simulated subject learned. In contrast, SCODEL-abstractive and SCODEL-attentive paid different amounts of attention to the feature dimensions, with notable distinction in the degree of difference that was produced accordingly; abstractive learners had greater differences (they were more likely to pay attention to either one of the two redundant dimensions), whereas attentive learners allocated similar amounts of attention to the redundant (and other) dimensions, resulting in smaller differences.

In sum, incorporating stochastically driven hypothesistesting-type learning with different objectives, ALCOVE successfully reproduced several different types of hypothetical, yet realistic, learners, as well as more realistic individual differences in attention allocation patterns.

\section{Simulation 2: Learning Via Supervision and Communication}

In the present study, members of pairs of strict errorminimizing learners (i.e., the EMN function) simultaneously learned to categorize stimuli together through both supervised learning and communication with each other. That is, after each training epoch, each learner calculated the utility index consisting of categorization accuracy (Equation 13) and the communicative index (Equation 25). Subsequently, on the basis of the utility value, each of the

Table 3

Squared Differences in the Amounts of Attention Allocated to Feature Dimensions 1 and 2

\begin{tabular}{lccc}
\hline \multicolumn{1}{c}{ Model } & Maximum & Mean & SD \\
\hline Abstractive SCODEL & 0.614 & 0.186 & 0.184 \\
Attentive SCODEL & 0.178 & 0.013 & 0.024 \\
Standard ALCOVE & 0.000 & 0.000 & 0.000 \\
\hline
\end{tabular}


two learners independently accepted or rejected the new coefficient configurations.

Method. The details of the simulation method were the same as those in Simulation 1. The communicative index, defined as in Equation 25, was used to adjust the compatibility of category knowledge, and the error minimization objective function (i.e., Equation 13) was used to evaluate the individuals' sets of hypotheses. Thus, the grand utility function was defined as

$$
\begin{aligned}
Z(\theta)= & \sum_{n}^{N} \sum_{k}^{K}\left(d_{k}-O_{k}\right)^{2}+\gamma_{w} \sum_{k}^{K} \sum_{j}^{J}\left(w_{k j}^{*}-w_{k j}\right)^{2} \\
& +\gamma_{\alpha} \sum_{i}^{I}\left(\frac{\alpha_{i}^{*}}{\sum_{m=1}^{I} \alpha_{m}^{*}}-\frac{\alpha_{i}}{\sum_{m=1}^{I} \alpha_{m}}\right)^{2} .
\end{aligned}
$$

There was a total of 100 pairs of simulated subjects trained with 500 training blocks. For a comparative purpose, another 100 pairs were trained without communicating with each other. The $\gamma$ coefficients were selected arbitrarily for the communicating pairs $\left(\gamma^{w}=1.5\right.$ and $\gamma^{\alpha}=$ 1.5 for communicating pairs); for the noncommunicating pairs, the coefficients were fixed at zero in order to prohibit them from communicating. Except for the $\gamma$ coefficients, exactly the same model settings were used for the pairs with and without communication.

Results. Table 4 shows the results of Simulation 2, based on the 100 pairs learned with communication and supervision and another 100 pairs learned with supervision only. Both types of learners were able to learn with approximately identical accuracy. However, only for the pairs whose members communicated during training was there a similar category structure or coefficient configuration, as suggested by mean correlation coefficients.

\section{Simulation 3: Inactive Exemplars}

The majority of exemplar-theory-based computational models of categorization and category learning assume that all exemplars form association with category nodes (e.g., Kruschke, 1992; Kruschke \& Johansen, 1999). However, such usage of limited mental recourses sounds inefficient and, perhaps, implausible, particularly when there are many distinctive yet very similar exemplars. This characteristic of exemplar-based models has been a source of criticism and debate about their descriptive validity (e.g., Love et al., 2004). The purpose of the pres-

Table 4

Results of Simulation 2: Mean Categorization Accuracies and Mean Correlation Coefficients for Paired Learners' Category Knowledge

\begin{tabular}{lcc}
\hline & With & \\
& Communication & $\begin{array}{c}\text { Without } \\
\text { Communication }\end{array}$ \\
\hline Accuracy & .927 & .936 \\
Mean CORR $(\alpha)$ & .756 & .094 \\
Mean CORR $(w)$ & .912 & .085 \\
\hline
\end{tabular}

ent study is not to criticize or reevaluate the exemplar theory. Rather, it is meant to show that the processes and outcomes of SCODEL's context-dependent learning can provide better qualitative interpretations of the utilization of exemplars. In particular, if we can assume that people implicitly or explicitly try to learn categories with a smaller amount of effort (i.e., Equation 14), a SCODELtype learner forms association links between category labels and a smaller number of exemplars, utilizing a smaller number of similar yet distinctive exemplars. An exemplar without an association link will be termed an inactive exemplar hereafter. Note that even without an association link, an inactive exemplar can be correctly categorized if there is a similar exemplar with the correct association link. This type of exemplar utilization appears to be more realistic than the utilization assumed and adapted by the previous models of categorization on the basis of the exemplar theory.

In the present study, the abstractive SCODEL and the standard ALCOVE were trained to learn simple categories, where there are 25 similar yet distinctive exemplars in each category. Then the number of exemplars without any "significant" association links with category nodes was analyzed.

Stimuli. A simple two-dimensional, two-category, 50exemplar (25 per each category) stimulus set was created for Simulation 3 (Figure 2). For both categories, Dimension 1 was the only relevant dimension (i.e., Category $=$ $\mathrm{A}$ if $\mathrm{D} 1 \approx 1$ and Category $=\mathrm{B}$ if $\mathrm{D} 2 \approx 0$ ). A small noise drawn randomly from the Gaussian distribution, with its standard deviation equal to 0.1 , was added to each instance's Dimension 1 value. Dimension 2 was irrelevant, and its values were drawn randomly from the uniform distribution.

Method. The details of the simulation method were the same as those in Simulation 1. There was a total of 100 abstractive learners in the present simulation study. The subset selection function was used for the association weights (Equation 19A with $\zeta=0.1$ ), and no regularization was imposed on the attention coefficients, since they were not the foci of the present simulation study. The $\gamma$ coefficient for association weights was fixed at 0.1 . For the standard gradient-based ALCOVE, as in Simulation 1, the parameter values were selected randomly. The ranges of the parameters were [0.1 10] for $c$ and $\phi$ and [0.001 1] for the two learning rates. There were a total of 10,000 standard ALCOVE learners. The main reasons for the difference in the numbers of simulated subjects is that whereas, due to its stochastic nature, SCODEL sufficiently simulates several different types of learners with the same parameter configuration, ALCOVE in general requires manipulations of its model parameters to simulate various types of learners. For both models, all the simulated learners were screened for their categorization accuracies at $75 \%$. This was done to prevent the learners from forming inactive exemplars by not learning. An inactive exemplar is defined as an exemplar whose maximum association weight to any category node is less than 0.1 . 


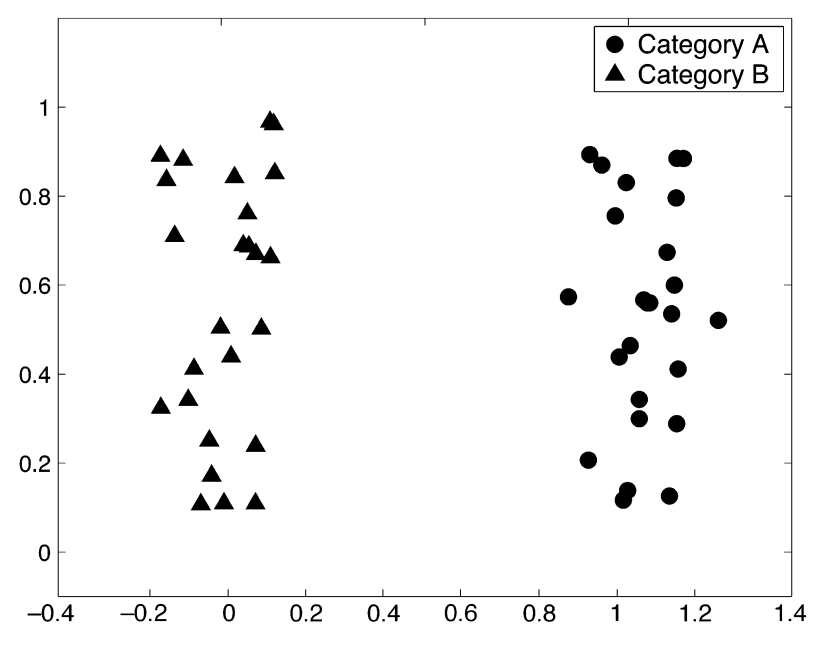

Figure 2. The stimulus set used in Simulation 3.

Results and Discussion. Nine SCODEL and 1,212 ALCOVE learners were replaced on the basis of the screening criterion. There was no single standard ALCOVE learner having any inactive exemplars. In other words, the ALCOVE learners learned to form association between all 50 exemplars to at least one category node and utilized all memorized exemplars in categorizing each stimulus. In contrast, on average, SCODEL learners had 32 inactive exemplars, with the minimum and maximum being 21 and 41, respectively (see Figure 3). Although the SCODEL prediction seems more plausible than that of the standard ALCOVE, for this simple two-class categorization task, the utilization of 18 exemplars by SCODEL also appears to be unrealistic to some extent. Conceivably, there might have been better parameter configurations that could result in larger num-

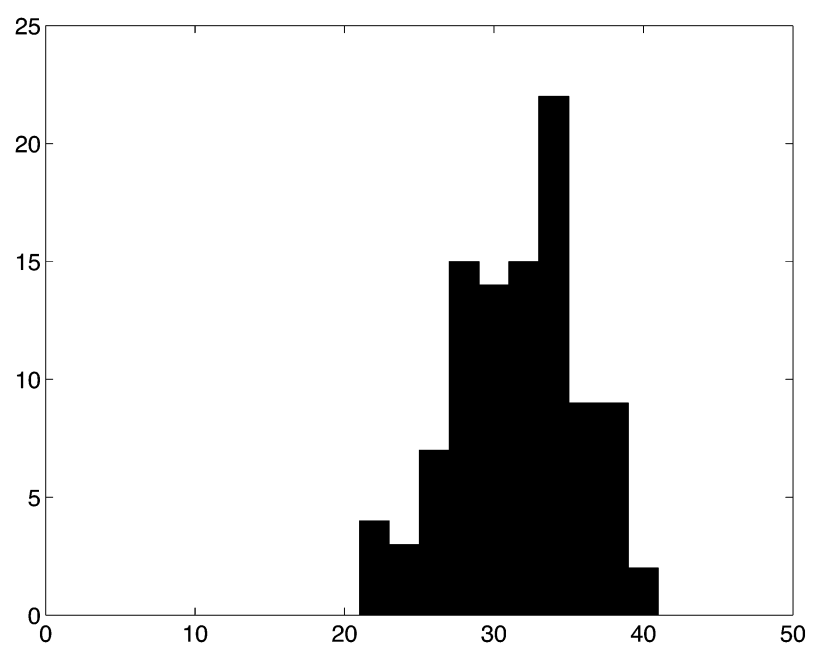

Figure 3. The distribution of the numbers of inactive exemplars resulted in SCODEL-type learning in Simulation 3. bers of inactive exemplars. Another, perhaps more qualitatively descriptive way to achieve larger numbers of inactive exemplars is to make regularization processes dynamic (e.g., by dynamically changing gamma coefficients). Dynamic regularization processes sound promising. But since this is beyond the scope of the present study, it will not be modeled in this article.

\section{Simulation 4: Simulation of an Empirical Study}

In the present simulation study, SCODEL's predictions were compared with real human behaviors. To do this, I simulated the results of an empirical study on classification learning: Study 2 in Matsuka (2002; also reported in Matsuka \& Corter, 2003). In this study, there were two perfectly redundant feature dimensions, Dimension 1 and Dimension 2 (see Table 5), which were also perfectly correlated with category membership. Thus, information from only one of the two correlated dimensions was necessary and sufficient for perfect categorization performance. Besides classification accuracy, data on the amount of attention allocated to each feature dimension were collected in the empirical study. The measures of attention used were based on feature-viewing time, as measured in a MouseLab-type interface (Bettman, Johnson, Luce, \& Payne, 1993) that can be considered to be a software version of an eyetracking device.

The empirical results that I tried to simulate indicated that 13 out of 14 subjects were able to categorize the stimuli almost perfectly. The aggregated results suggested that, on average, the subjects paid attention to both of the redundant dimensions approximately equally (Figure 4, top row, middle column). However, more interestingly, when the attention data were analyzed per individual, it was found that many of the subjects tended to pay attention primarily to only one of the two correlated dimensions, particularly in the late learning blocks, as is shown in Figure 4, top right column (Matsuka \& Corter, 2003). The scatterplot shows the relative amounts (thus, the sum of the amount of attention allocated across all dimensions is fixed at 1) of attention allocated to Feature Dimension 1 (on the $x$-axis) and Dimension 2 (on the $y$-axis). This suggests that the subjects might have tried to perform the categorization task with smaller amounts of mental effort, attending to a smaller number of feature dimensions.

Method. There were two SCODEL-type learners in the present simulation study - namely, abstractive SCODEL (abSCODEL) and error-minimizing SCODEL (emSCODEL). The standard ALCOVE was not evaluated in the present simulation study, because its standard gradient learning method had been shown to be unsuccessful in replicating individual differences when attention allocation was initialized equally, as has been shown in the Discussion section for Simulation 1. In addition, the incapability of the standard ALCOVE with gradient descent learning to replicate differential usage of the redundant dimensions was shown in a simulation study conducted by Matsuka and Corter (2004). 
Table 5

The Abstract Category Structure Used in

Simulation 4 (Matsuka, 2002)

\begin{tabular}{ccccc}
\multicolumn{5}{c}{ Simulation 4 (Matsuka, 2002) } \\
\hline & \multicolumn{4}{c}{ Dimension } \\
\cline { 2 - 5 } Category & 1 & 2 & 3 & 4 \\
\hline A & $1^{*}$ & $1^{*}$ & 3 & 4 \\
A & $1^{*}$ & $1^{*}$ & 4 & 1 \\
A & $1^{*}$ & $1^{*}$ & 1 & 2 \\
B & $2^{*}$ & $2^{*}$ & 2 & 1 \\
B & $2^{*}$ & $2^{*}$ & 3 & 2 \\
B & $2^{*}$ & $2^{*}$ & 4 & 3 \\
C & $3^{*}$ & $3^{*}$ & 1 & 3 \\
C & $3^{*}$ & $3^{*}$ & 2 & 4 \\
C & $3^{*}$ & $3^{*}$ & 3 & 1 \\
D & $4^{*}$ & $4^{*}$ & 4 & 2 \\
D & $4^{*}$ & $4^{*}$ & 2 & 3 \\
D & $4^{*}$ & $4^{*}$ & 1 & 4 \\
\hline
\end{tabular}

*Diagnostic feature.

Both models were run in a simulated training procedure in which the correct classification responses for the stimuli of the experiment were learned. They were run for 500 blocks of training, where each block consisted of a complete set of the training instances. For each model, the final results were based on 100 replications. The model configurations for abSCODEL and emSCODEL were the same, except for the additional coefficient penalization functions incorporated into abSCODEL to model abstraction processes in category learning. Their feedforward parameters, specificity parameter $c$ and decisiveness parameter $\phi$, were set to 5 and 3 , respectively, for both types of learners. The random numbers used for coefficient updates for these two models were drawn from the Cauchy distribution, and the random number generation algorithm and the temperature-decreasing functions were based on Ingber (1989), as in the previous three simulation studies. For abSCODEL, the ridge penalty (Equation 18A) was imposed on the association weights, and a subset selection method (Equation 19B with $\zeta=0.1$ ) was used for the relative attention strengths. The $\gamma$ coefficients were 0.1 for both association weights and attention coefficients.

Results. Both abSCODEL and emSCODEL were able to categorize the stimuli successfully. The average categorization accuracies were $92.1 \%$ and $94.9 \%$ for abSCODEL and emSCODEL, respectively. Both models successfully replicated aggregated or averaged relative attention allocations to the four feature dimensions (Figure 4, middle column). Qualitatively, abSCODEL appears to have been more successful in replicating people's not paying attention to both Dimensions 1 and 2 equally, whereas emSCODEL overestimated the number of people who would pay attention to both Dimensions 1 and 2. The results may indicate that the subjects in Matsuka's (2002) study either explicitly or implicitly tried to learn the stimuli-category relationship with a smaller amount of effort. In other words, while they were learning about stimuli, they regularized their knowledge of categories so as to be compact and (acceptably) accurate.
Such processes are clearly effective in many real-world categorization contexts and are probably true for the majority of categorization tasks in real life.

\section{GENERAL DISCUSSION}

Gradient descent has been a de facto standard learning algorithm in computational models of category learning. However, there have been three concerns associated with its descriptive validity - namely, complexity, regularity, and context independency. To account for these difficulties, the present study introduced an alternative learning algorithm family with emphasis on modeling simple, individually varying, and context-dependent learning processes. In particular, the latter constitutes an innovation in the field; to the best of the author's knowledge, this is the first attempt at designing a category-learning model specifically tuned into context-dependent demands and constraints. A series of simulation studies showed that the present model, termed SCODEL, has the competence to replicate several different types of learners in hypothetical yet realistic situations. In addition, although it is not documented in the present study, the stochastic learning model (Matsuka \& Corter, 2004), which can be considered to be a special case of SCODEL, can reproduce insightful or all-or-none learning curves for both categorization accuracies and attention distribution.

\section{Future Directions}

One apparent limitation of the present research is a lack of empirical evidence supporting the validity of SCODEL (see, however, Matsuka \& Corter, 2004). That is, only one simulation study was concerned with actual human data. Comparisons of SCODEL's predictions and empirical data would certainly be helpful in evaluating its effectiveness. In particular, richer empirical data on categorization, including classification response, attention learning (Corter \& Matsuka, 2004; Matsuka, 2002; Rehder \& Hoffman, 2003), and stimulus recognition (Sakamoto \& Love, 2004) with manipulation of contextual factors should be collected. Another possibly interesting research direction is to apply the SCODEL framework to a broader range of learning models, in order to see how generalizable it is as a model of human learning.

\section{RULEX}

One unique model of categorization in which learning algorithms other than gradient methods are utilized is RULEX (Nosofsky, Palmeri, \& McKinley, 1994). In particular, it incorporates a sequential hypothesis-testing-like learning algorithm. In its first stage of learning, RULEX tries to identify a categorization rule defined by a single perfectly predictive feature dimension, and the search process continues until all the feature dimensions are tested. In the second stage, it searches for (multiple) imperfect single-dimension rules, followed by conjunctive rules in the third stage. Although, RULEX sounds plausible and could replicate individual differences for several stimu- 


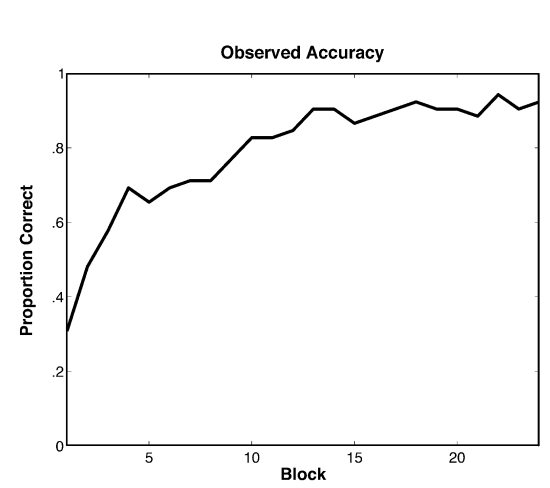

\section{Observed Empirical Results}
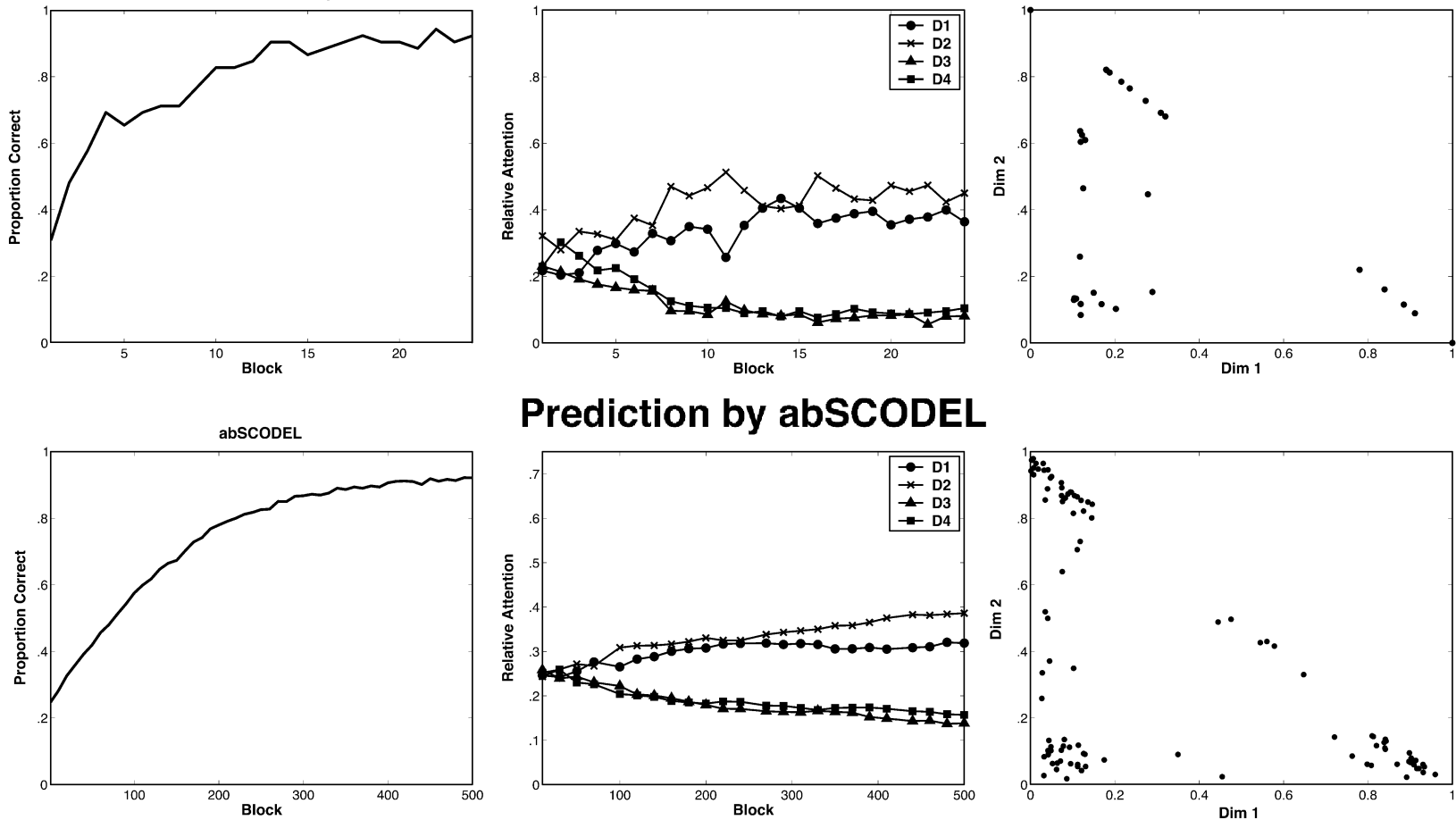

Prediction by abSCODEL
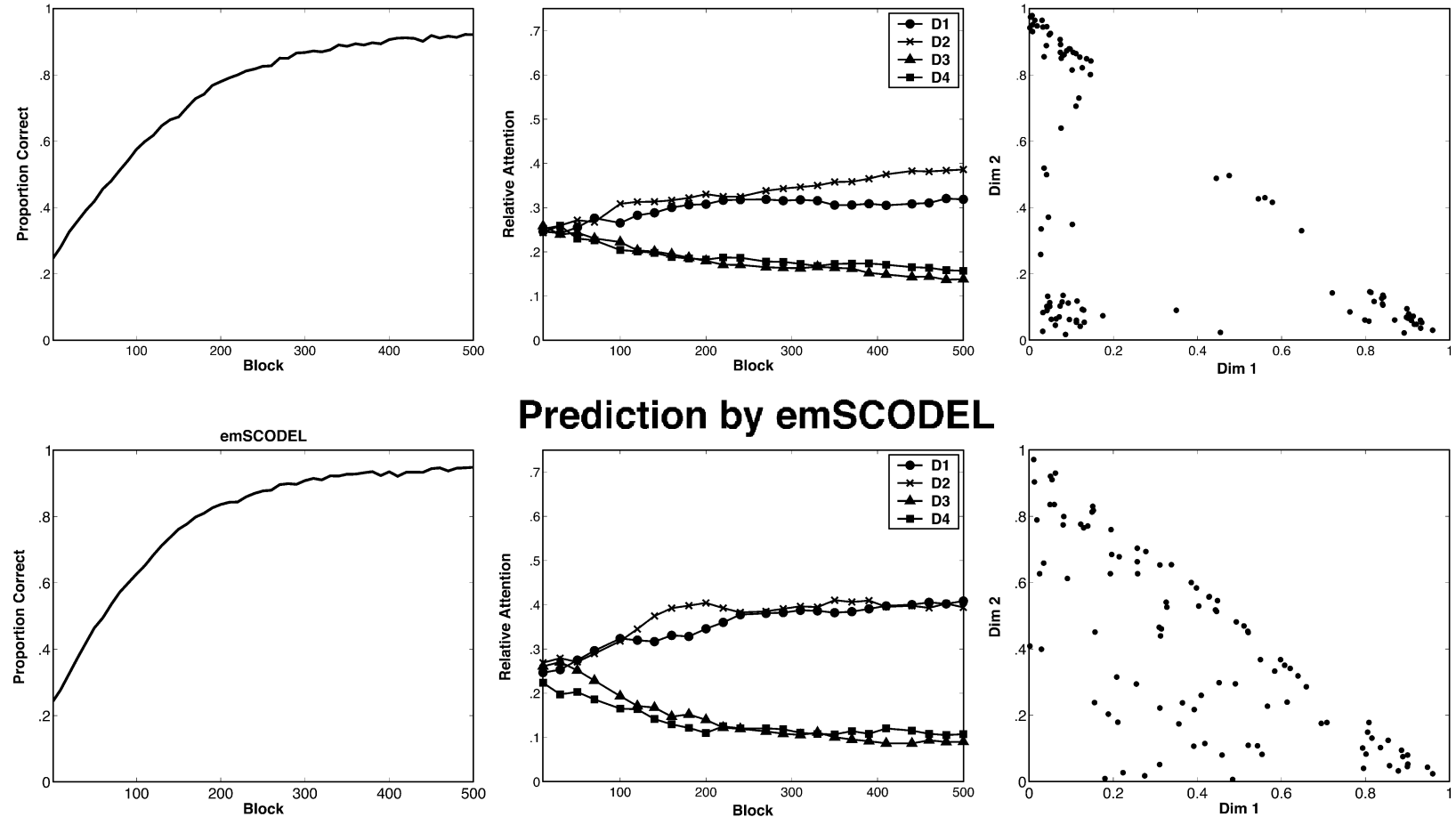

Prediction by emSCODEL
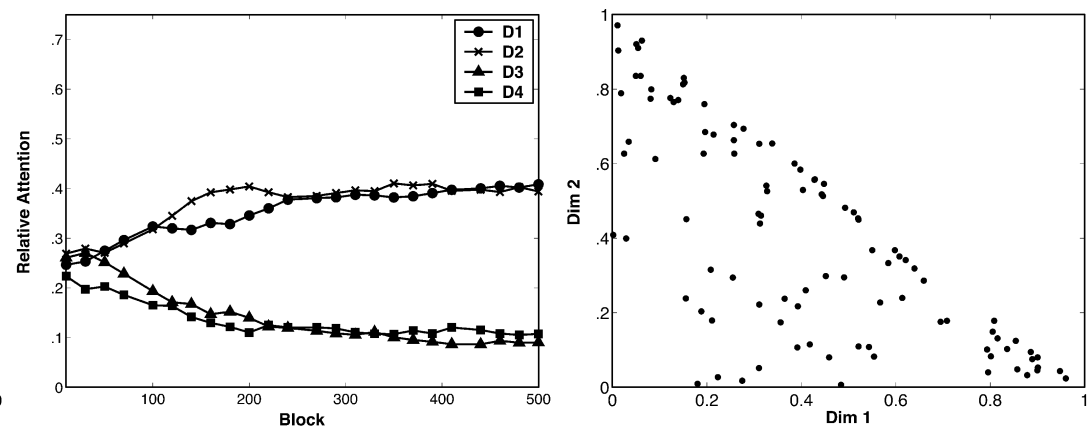

Figure 4. Results of Simulation 4. Top row: observed empirical results of Matsuka (2002). Middle row: predictions by abstractivelearning SCODEL. Bottom row: predictions by error-minimizing SCODEL. The graphs in the left column show classification accuracies. The graphs in the middle column show the amounts of relative attention allocated to the four feature dimensions. The scatterplots in the right column compare relative attention allocated to Dimensions 1 and 2 for the last three blocks, where each dot represents an observation.

lus sets, it may be considered to be more normative than descriptive of real humans in terms of efficiency in information usage. That is, RULEX predicts that people are always capable of identifying the minimal and sufficient number of diagnostic feature dimensions and only use exactly the same diagnostic dimensions once they have been learned.

SCODEL's take-all-or-none coefficient-updating strategy may be considered to be a type of hypothesis-testing learning model, which makes it similar to RULEX. However, its random search method, interpreted as unstructured hypothesis generation and search, is very distinct from RULEX, whose hypothesis generation algorithm is very strategic and well structured. SCODEL can pay attention to any number of feature dimensions, as long as the coefficient configurations result in the acceptable categorization accuracies. Thus, the stochastic learning methods can result in paying attention to irrelevant dimensions, as long as its coefficient configuration is associated with good classification accuracy. In other words, when there are several minima, which is probably true for real-world category-learning tasks, stochastic learning can result in several different learning trajectories and coefficient configurations (i.e., association weight and attention allocation), corresponding to possible individual differences. In contrast, RULEX would predict 
that people always pay attention to the least number of dimensions throughout the entire learning phase (i.e., if there was only one diagnostic dimension, it would never pay attention to more than one dimension during its entire learning process, because it starts by making the hypothesis that only a single dimension is diagnostic), which may be too normative a prediction in terms of the efficiency of information usage. If it were trained to categorize Matsuka's (2002) stimulus set, RULEX, in its original form, would predict that every simulated subject will pay attention exclusively to only one dimension of the two diagnostic correlated dimensions, resulting in a much more efficient usage of diagnostic information than that shown by the subjects in the study.

\section{Extension}

SCODEL's regularization process is static. However, people's regularized learning processes are probably dynamic. For example, Johansen and Palmeri (2002) suggested that there are representational shifts during category learning, where people tend to form a rule-like representation at first and then an exemplar-like representation in the later learning stages. This phenomenon may be caused by the fact that people try to form compact yet sufficiently accurate concepts by creating "rules" in the beginning and then, once such processes have been executed successfully (which frees up computational power of the brain), they try to form expert-like knowledge about particular category domains by remembering exemplars. If this hypothesis is valid, modifying SCODEL to account for a dynamic regularization process may make it capable of replicating the phenomenon. Although it seems that great care and meticulous thought are necessary to make SCODEL dynamic, the modification would be a constructive step toward plausibly and descriptively modeling human category-learning processes.

\section{REFERENCES}

Anderson, J. R. (1990). The adaptive character of thought. Hillsdale, NJ: Erlbaum.

Ashby, F. G., Alfonso-Reese, L. A., Turken, A. U., \& Waldron, E. M. (1998). A neuropsychological theory of multiple systems in category learning. Psychological Review, 105, 442-481.

Bettman, J. R., Johnson, E. J., Luce, M. F., \& Payne, J. W. (1993). Correlation, conflict, and choice. Journal of Experimental Psychology: Learning, Memory, \& Cognition, 19, 931-951.

Bower, G. H., \& Trabasso, T. R. (1963). Reversals prior to solution in concept identification. Journal of Experimental Psychology, 66, 409418.

Cherkassky, V., \& Mulier, F. (1997). Learning from data: Concepts, theory, and methods. New York: Wiley.

Corter, J. E., \& MATsukA, T. (2004, November). Empirical measures of attention allocation in classification learning: A replication of Medin and Schaffer (1978). Paper presented at the 45th Annual Meeting of the Psychonomic Society, Minneapolis.

Feldman, J. (2003). The simplicity principle in human concept learning. Current Directions in Psychological Science, 12, 227-232.

HAYKIN, S. (1999). Neural networks: A comprehensive foundation (2nd ed.). Upper Saddle River, NJ: Prentice-Hall.

INGBER, L. (1989). Very fast simulated re-annealing. Journal of Mathematical Computer Modelling, 12, 967-973.
Johansen, M. K., \& PAlmeri, T. J. (2002). Are there representational shifts during category learning? Cognitive Psychology, 45, 482-553. Johnson, K. E., \& Mervis, C. B. (1997). Effects of varying levels of expertise on the basic level of categorization. Journal of Experimental Psychology: General, 126, 248-277.

Kosslyn, S. M., Alpert, N. M., \& Thompson, W. L. (1995). Identifying objects at different levels of hierarchy: A positron emission topography study. Human Brain Mapping, 3, 107-132.

KruschKe, J. K. (1992). ALCOVE: An exemplar-based connectionist model of category learning. Psychological Review, 99, 22-44.

KruschKe, J. K., \& Johansen, M. K. (1999). A model of probabilistic category learning. Journal of Experimental Psychology: Learning, Memory, \& Cognition, 25, 1083-1119.

Love, B. C. (2003). The multifaceted nature of unsupervised category learning. Psychonomic Bulletin \& Review, 10, 190-197.

Love, B. C., Medin, D. L., \& Gureckis, T. M. (2004). SUSTAIN: A network model of category learning. Psychological Review, 111, 309-332

Markman, A. B., \& Gentner, D. (1996). Commonalities and differences in similarity comparisons. Memory \& Cognition, 24, 235-249.

Markman, A. B., \& MaKin, V. S. (1998). Referential communication and category acquisition. Journal of Experimental Psychology: General, 127, 331-354.

Matsuka, T. (2002). Attention processes in computational models of category learning. Unpublished doctoral dissertation, Columbia University.

MATSUKA, T. (2004). Biased stochastic learning in computational model of category learning. In R. Alternman \& D. Kirsh (Eds.), Proceedings of the 26th Annual Meeting of the Cognitive Science Society (pp. 915920). Mahwah, NJ: Erlbaum.

MatsuKa, T. (2005). Modeling human learning as context-dependent knowledge utility optimization. In L. Wang, K. Chen, \& Y. Ong (Eds.), International Conference on Natural Computation (LNCS3610, pp. 933-946). Berlin: Springer-Verlag.

Matsuka, T., \& CoRTER, J. E. (2003, November). Empirical studies of attention processes in category learning. Poster presented at the 44th Annual Meeting of the Psychonomic Society, Vancouver.

Matsuka, T., \& CoRTER, J. E. (2004). Stochastic learning algorithm for modeling human category learning. International Journal of Computational Intelligence, 1, 40-48.

Matsuka, T., Corter, J. E., \& Hanson, S. J. (2004). Irresistibly attractive fruitless feature dimensions. In M. Lovett, C. Schunn, C. Lebière, $\&$ P. Munro (Eds.), Proceedings of the 6th International Meeting of Cognitive Modelling (pp. 370-371). Mahwah, NJ: Erlbaum.

Matsuka, T., Corter, J. E., \& Markman, A. B. (2003). Attention learning in adaptive network models of categorization. Manuscript submitted for publication.

Medin, D. L., \& Schaffer, M. M. (1978). Context theory of classification learning. Psychological Review, 85, 207-238.

Metropolis, N., Rosenbluth, A. W., Rosenbluth, M. N., TellER, A. H., \& TELLER, E. (1953). Equation of state calculations by fast computing machines. Journal of Chemical Physics, 21, 1087-1092.

Mitchell, T. M. (1997). Machine learning. Boston: McGraw-Hill.

NosofSKy, R. M. (1986). Attention, similarity and the identificationcategorization relationship. Journal of Experimental Psychology: General, 115, 39-57.

Nosofsky, R. M., Gluck, M. A., Palmeri, T. J., McKinley, S. C., \& Glauthier, P. (1994). Comparing models of rule-based classification learning: A replication and extension of Shepard, Hovland, and Jenkins (1961). Memory \& Cognition, 22, 352-369.

Nosofsky, R. M., Palmeri, T. J., \& MCKinley, S. C. (1994). Rule-plusexception model of classification learning. Psychological Review, 101, 53-79.

ReHDER, B., \& HofFman, A. B. (2003). Eyetracking and selective attention in category learning. In R. Alternman \& D. Kirsch (Eds.), Proceedings of the 25th Annual Meeting of the Cognitive Science Society (pp. 976-981). Mahwah, NJ: Erlbaum.

Rosch, E., Mervis, C. B., Gray, W. D., Johnson, D. M., \& BoyesBraem, P. (1976). Basic objects in natural categories. Cognitive Psychology, 8, 382-439. 
SaKamoto, Y., \& Love, B. C. (2004). Schematic influences on category learning and recognition memory. Journal of Experimental Psychology: General, 133, 534-553.

TANAKA, J., \& GAUTHIER, I. (1997). Expertise in object and face recognition. In R. L. Goldstone, P. G. Schyns, \& D. L. Medin (Eds.), Perceptual learning: The psychology of learning and motivation (Vol. 36, pp. 83-125). San Diego: Academic Press.

TANAKA, J., \& TAYLOR, M. (1991). Object categories and expertise: Is the basic level in the eye of the beholder? Cognitive Psychology, 23, 457-482.

\section{NOTE}

1. There is a unique category-learning model called RULEX (Nosofsky, Palmeri, \& McKinley, 1994), also intended to model category learning at the individual level. However, RULEX was not considered in the present research, because the main theme of the present research is to introduce a family of general learning models and to contrast it with another general learning method - namely, gradient descent—but not to compare particular category-learning models.

(Manuscript received November 15, 2004;

revision accepted for publication March 31, 2005.) 